\title{
Avaliação de Melhorias em Processos de Software Durante a Execução de um Projeto
}

\author{
Mylene Cabral $^{1}$, Ana Regina Rocha ${ }^{1}$, Gleison Santos ${ }^{2}$ \\ ${ }^{1}$ Programa de Engenharia de Sistemas e Computação - COPPE/UFRJ \\ Caixa Postal 68.511 - CEP 21.945-970 - Rio de Janeiro - RJ - Brasil \\ ${ }^{2}$ Programa de Pós-Graduação em Informática - UNIRIO \\ Av. Pasteur 458, Urca - CEP 22290-240 - Rio de Janeiro - RJ - Brasil \\ mylene@gmail.com, darocha@cos.ufrj.br, gleison.santos@uniriotec.br
}

\begin{abstract}
This paper presents proposal to support the evaluation of changes in the process defined for an ongoing project supported by multicriteria decision techniques. We expect that members of the Software Engineering Process Group of an organization are able to evaluate, through a method to support decision making, whether is advantageous to implement the improvements undertaken in the standard process in the defined process of an ongoing project. The proposed approach was evaluated through a case study performed in three software development organizations. The results indicate that the approach is useful and easy to use.
\end{abstract}

Resumo. Este trabalho apresenta uma proposta de apoio à avaliação de alterações no processo definido para um projeto que se encontra em execução por meio de análise decisória multicriterial. Espera-se, assim, que membros do Grupo de Processos de uma organização de desenvolvimento de software sejam capazes de avaliar, por meio de um método de apoio à tomada decisão, se é vantajoso implantar as melhorias realizadas no processo padrão no processo definido para um projeto já em execução. A proposta foi avaliada por meio de estudo de caso executado em três organizações de desenvolvimento de software. Os resultados dos estudos revelaram indícios de que a proposta é útil e de fácil utilização.

\section{Introdução}

Melhorias em processos podem ocorrer em dois níveis: (i) organizacional, quando a melhoria é implementada nos processos padrão da organização; e (ii) de projeto, quando a melhoria é implementada no processo definido para os projetos em execução. Independente do nível, as melhorias precisam ser implementadas de forma controlada. Além disso, é importante que os efeitos da mudança sejam avaliados antes que ela seja efetivada [Raffo, 2005]. O uso de pilotos para avaliar a efetividade da proposta de melhoria é sugerido por modelos de referência, como CMMI [SEI, 2010] e MR-MPS-SW [SOFTEX, 2012]. Como os efeitos da mudança só podem ser avaliados após a conclusão do projeto, esta abordagem não é viável quando a alteração ocorre em processos definidos para projetos que já estão em execução.

Uma alternativa à adoção de pilotos pode ser a utilização de modelos de simulação de processos de software. No entanto, estudos destacam a dificuldade de se construir e manter estes modelos [Raffo et al., 2003; Park et al., 2007], visto que exigem alto 
grau de conhecimento relacionado não só a modelos de simulação, mas também ao processo de desenvolvimento a ser modelado. Técnicas de análise multivariada de dados também podem ser utilizadas para predizer os efeitos de mudanças em processos [Hair et al., 2007]. Porém, para que o erro da predição seja reduzido, é necessária uma boa base de dados históricos de execuções dos processos, o que pode ser um empecilho para a maioria das organizações.

A implementação de melhorias em processos de software pode ser considerada um problema de tomada de decisão. Assim, é possível adotar métodos multicritério de apoio à decisão (AMD) para avaliar qual é o melhor processo padrão para a organização ou o melhor processo definido para um projeto em execução. A vantagem desses métodos é que a execução não exige experiência além da que já é exigida ao papel que o decisor desempenha na organização. No entanto, a seleção do método AMD mais apropriado para o problema de decisão tem um papel crucial no sucesso do processo de tomada de decisão [Al-Shemmeri et al., 1997; Guitouni e Martel, 1998].

Diante desse contexto, este trabalho propõe uma abordagem para apoiar a avaliação prévia da alteração no processo definido para um projeto que se encontra em execução, baseada em análise decisória multicriterial. Espera-se, desta forma, que seja possível avaliar se é vantajoso estender as melhorias implementadas no processo padrão de desenvolvimento de software da organização aos processos definidos para os projetos que já se encontram em execução, evitando ou reduzindo, assim, as chances de ocorrência de efeitos contrários ao que é esperado.

O presente artigo está organizado em cinco seções, incluindo esta introdução. A Seção 2 apresenta os trabalhos relacionados ao apoio a avaliação de melhorias em processos em execução. A Seção 3 trata de apoio multicritério à tomada de decisão. A Seção 4 descreve a abordagem proposta. Na Seção 5 são apresentados os três estudos de casos executados para avaliar a abordagem. Por fim, na Seção 6, são apresentadas as considerações finais e trabalhos futuros.

\section{Trabalhos Relacionados}

Apesar da importância de se avaliar os efeitos de uma melhoria antes de efetivá-la [Raffo, 2005], um estudo de mapeamento sistemático da literatura, detalhado em [Cabral, 2012], observou que apenas duas das abordagens para melhorias em processos durante a execução de um projeto se propõem a apoiar a avaliação do impacto da mudança no processo antes de implementá-la. São elas: PIE (Process Instance Evolution) [Beydeda e Gruhn, 2004] e PROMPT (PROject Management of Process Tradeoffs) [Raffo, 2005].

PIE (Process Instance Evolution) [Beydeda e Gruhn, 2004] é um sistema para avaliar o impacto de evoluções dinâmicas de processo de software com ênfase em aspectos de apoio à tomada de decisão. A técnica proposta faz uso de um algoritmo capaz de extrair informações a partir do cronograma do processo, que consiste de itens que indicam o fim da execução do processo. Cada item possui atributos para custo total e probabilidade de concluir no tempo planejado. O cronograma para um processo específico pode ser utilizado para determinar a distribuição de probabilidade tanto de duração como de custo total. Uma vez identificada a distribuição de probabilidade, medidas estatísticas, como, por exemplo, valor esperado e variância, podem ser utilizadas como medidas de risco. Baseado em tais medidas de risco, uma função de utilidade para cada alternativa pode ser determinada, levando em consideração as preferências e aversão a 
risco do gerente de processo. Desta forma, selecionar uma alternativa envolve computar a função de utilidade para todas as alternativas disponíveis e selecionar aquela que domina todas as outras.

O sistema PIE [Beydeda e Gruhn, 2004] espera que a organização possua uma boa quantidade de dados históricos de execução de seus processos de modo a tornar mais precisa a geração das distribuições de probabilidade e o cálculo das medidas de risco para os dois únicos parâmetros observados: duração e custo. Esta pré-determinação dos parâmetros também pode ser considerada uma limitação do trabalho, visto que os parâmetros de comparação das alternativas de projetos podem ser dos mais variados, dependendo dos objetivos estratégicos da organização ou, até mesmo, dos objetivos específicos do projeto.

O framework PROMPT (PROject Management of Process Tradeoffs) [Raffo, 2005] utiliza OBLC (Outcome Based Control Limits), funções de utilidade e medidas de desempenho financeiro. $\mathrm{Na}$ fase de planejamento, um modelo de simulação quantitativo auxilia na tomada de decisão, prevendo o desempenho de cada alternativa de processo. Durante a execução do projeto, dados atualizados alimentam parâmetros do modelo, provendo uma previsão mais precisa da trajetória atual e resultados esperados do projeto. Quando uma ação corretiva se torna necessária para tratar desvios no projeto, o modelo de simulação é utilizado para prever o desempenho das alternativas de processo em cada medida de desempenho, além de prever o risco ou variabilidade associada aos valores esperados. As distribuições previstas para cada alternativa de processo são comparadas aos OBCLs para determinar a probabilidade do desempenho do projeto ficar dentro dos limites de controle aceitáveis pelo gerente. Embora o framework PROMPT não apresente restrições quanto à quantidade de parâmetros a serem observados na comparação das alternativas de processo, é esperado que o usuário desenvolva um modelo de simulação. Estudos destacam a dificuldade de se construir e manter estes modelos [Raffo et al., 2003; Park et al., 2007], visto que, para isso, é necessário alto grau de conhecimento relacionado a modelos de simulação e ao processo de desenvolvimento a ser modelado.

\section{Apoio à Tomada de Decisão}

A tomada de decisão é o processo de escolher um curso de ação possível dentre várias alternativas [Malakooti, 1988]. Sabe-se, hoje em dia, que este processo vai além do modelo clássico: otimizar uma função de objetivo único visando encontrar uma "solução ótima" para um problema [Guitouni e Martel, 1998]. Acredita-se que aspectos conflitantes devem ser simultaneamente levados em consideração, de modo a estimular os decisores a considerarem não mais uma decisão ótima, mas uma decisão satisfatória [Guitouni e Martel, 1998; Brans, 2002; Forneck, 2008].

Guitouni e Martel [1998] afirmam que, apesar da grande quantidade de métodos AMD disponíveis, não existe um que retorne boas recomendações para todas as situações. Da mesma forma, Al-Shemmeri et al. [1997] acreditam que cada método AMD apresenta pontos fortes e pontos fracos que determinam em quais situações de tomada de decisão (DMS - Decision Making Situation) o método pode ou não ser utilizado. Como consequências da escolha inadequada de um método AMD pode-se considerar [Al-Shemmeri et al., 1997]: (i) a possibilidade de obtenção de resultados subótimos; (ii) perda de tempo e dinheiro decorrente do descarte de técnicas mais adequadas; e (iii) 
potencial desestímulo por parte dos tomadores de decisão com relação à aplicação da técnica.

Diante deste contexto, neste trabalho, houve uma preocupação com a seleção do método AMD mais adequado. Para tal, foi realizado um estudo em duas etapas. A primeira etapa foi conduzida com base em [Guitouni e Martel, 1998], que caracterizou 29 métodos AMD. A partir dessas caracterização, foi selecionado um subconjunto de métodos que apresentavam as características consideradas essenciais para tratar a situação de decisão em questão. Na segunda etapa, foram definidas novas características, não contempladas no trabalho de Guitouni e Martel [1998]. Cada método candidato foi cuidadosamente estudado para identificar estas novas características. O resultado do estudo identificou o método TOPSIS [Yoon e Hwang, 1995] como o mais apropriado. Detalhes deste estudo podem ser encontrados em [Cabral, 2012].

\subsection{O Método TOPSIS}

O método TOPSIS [Yoon e Hwang, 1995] é baseado no conceito de que a alternativa escolhida deve ter a menor distância da Solução Ideal e a maior distância da Solução Indesejável. A seguir são apresentados os passos do método (Quadro 1):

\section{Quadro 1. Passos do TOPSIS [Yoon e Hwang, 1995]}

Passo 1. Calcular matriz normalizada. A normalização por vetor é realiza por meio da Equação (1).

$$
r_{i j}=\frac{x_{i j}}{\sqrt{\sum_{i=1}^{m} x_{i j}^{2}}}
$$

Onde: $m$ é o número de alternativas e $-x_{i j}$ representa a pontuação do critério $j$ para a alternativa $i$.

Passo 2. Calcular matriz com os respectivos pesos. A pontuação normalizada com o peso é calculada da seguinte forma:

$v_{i j}=w_{j} r_{i j}$

Onde: $w_{j}$ é o peso definido para cada atributo ou critério

Passo 3. Identificar a Solução Ideal e a Solução Indesejada. $A^{*}$ e $A^{-}$são definidas em termos das pontuações normalizadas com seus pesos.

$$
\begin{aligned}
& A^{*}=\left\{v_{1}^{*}, v_{2}^{*}, \ldots, v_{n}^{*}\right\}=\left\{\left(\max v_{i j} \mid j \in J_{1}\right),\left(\min v_{i j} \mid j \in J_{2}\right)\right\} \\
& A^{-}=\left\{v_{1}^{-}, v_{2}^{-}, \ldots, v_{n}^{-}\right\}=\left\{\left(\min v_{i j} \mid j \in J_{1}\right),\left(\max v_{i j} \mid j \in J_{2}\right)\right\} \\
& \text { Onde: } J_{l} \text { é o conjunto de critérios de benefícios e } \mathrm{J}_{2} \text { é o conjunto de critérios de custo }
\end{aligned}
$$

Passo 4. Calcular medidas de separação. A distância entre as alternativas pode ser medida por meio da distância Euclidiana, calculada de acordo com a Equação (4):

$$
S_{i}^{*}=\sqrt{\sum_{j=1}^{n}\left(v_{i j}-v_{j}^{*}\right)^{2}} \quad S_{i}^{-}=\sqrt{\sum_{j=1}^{n}\left(v_{i j}-v_{j}^{-}\right)^{2}}
$$

Passo 5. Calcular similaridades com a Solução Ideal. O índice de similaridade é calculado a partir da seguinte fórmula:

$$
C_{i}^{*}=\frac{S_{i}^{-}}{\left(S_{i}^{*}+S_{i}^{-}\right)}
$$

$\mathrm{O}$ índice de similaridade pode variar de 0 a $1 . \mathrm{C}_{\mathrm{i}}^{*}=0$ significa que $\mathrm{A}_{\mathrm{i}}=\mathrm{A}^{-}$, isto é, a alternativa analisada é a Solução Indesejada. Em contrapartida, $C_{i}^{*}=1$ quer dizer que $A_{i}=A^{*}$, ou seja, a alternativa analisada é a Solução Ideal.

Passo 6. Classificar ordem de preferência. A alternativa com maior $C_{i}^{*}$ pode ser escolhida ou pode ser feita uma ordenação de todas as alternativas, de acordo com o $C_{i}^{*}$ calculado. 


\section{A Abordagem Proposta}

A proposta deste trabalho faz uso de dois processos: (i) um processo de tomada de decisão baseado no método de apoio à tomada de decisão selecionado TOPSIS, apresentado na seção 4.1; e (ii) um processo para melhorias em processos, apresentado na seção 4.2. Como o método TOPSIS não possui ferramenta própria, também foi elaborado um mecanismo de apoio desenvolvido a partir do Microsoft Excel® (Figura 1). São exibidos os mecanismos para estruturação das alternativas e critérios a serem considerados, a forma de avaliação das alternativas e as recomendações sugeridas pela aplicação do TOPSIS.
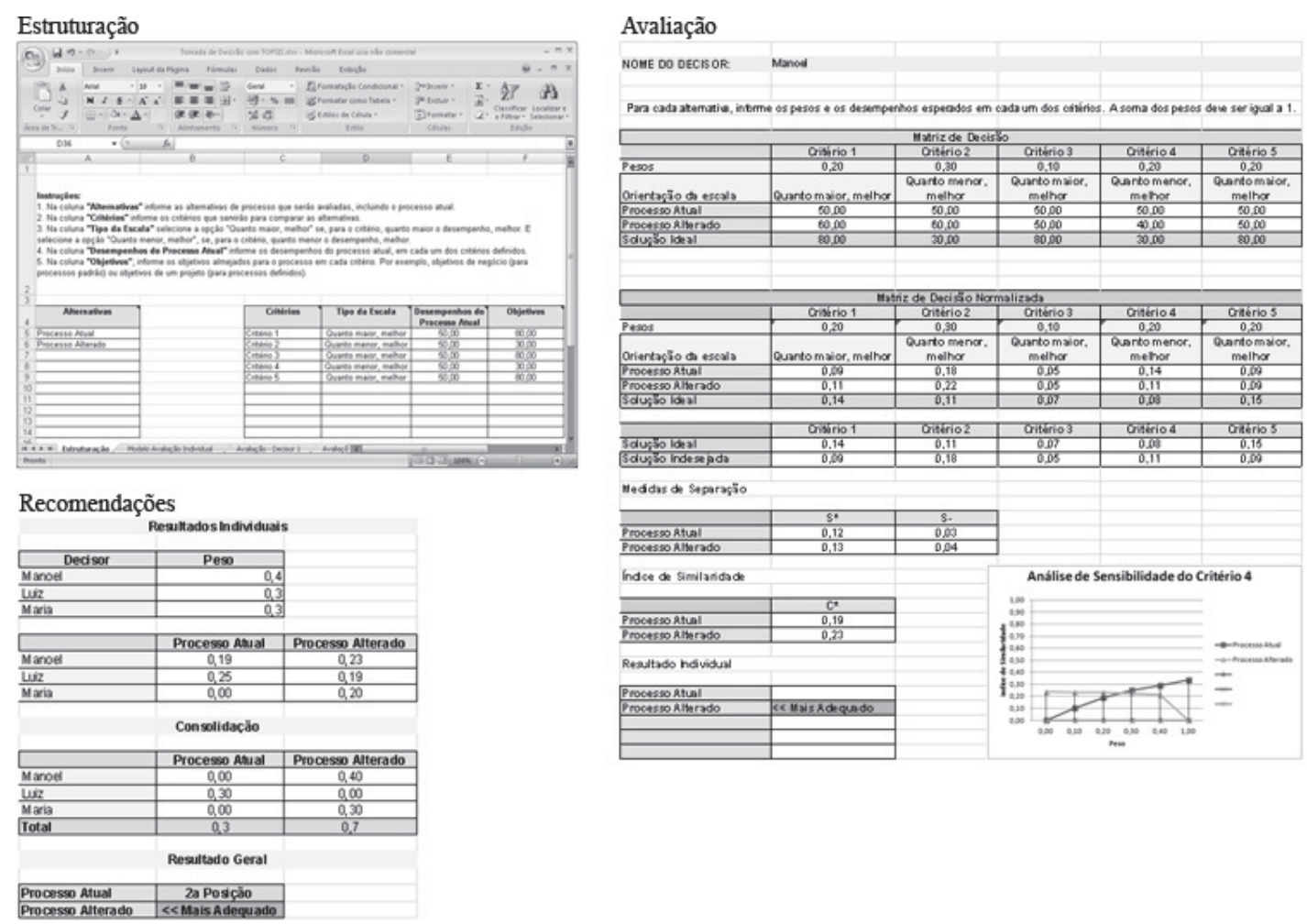

Figura 1. Mecanismo de apoio ao processo de tomada de decisão

\subsection{Processo de Tomada de Decisão para Melhoria em Processos}

Para tornar o método TOPSIS mais adequado ao problema em questão, foram definidas algumas adaptações. Baseado na proposta de Liou et al. [2011], a Solução Ideal passou a ser composta pelos desempenhos aspirados em cada alternativa e não mais pelos melhores desempenhos, como propõe a versão original do método. Essa adaptação foi considerada relevante, face à importância de se observar a proximidade das alternativas de processo com relação aos objetivos estratégicos da organização ou com relação aos objetivos do projeto.

Uma segunda adaptação foi a adoção de uma forma simplificada de condução de uma análise de sensibilidade dos pesos dos critérios, devido importância de verificar o quão sensível a pequenas variações é o modelo de decisão gerado, visto que a definição dos pesos dos critérios é feita com base na experiência do decisor.

A última adaptação foi a adoção de uma função de escolha social para que fosse possível levar em consideração a opinião individual de cada membro do Grupo de Processos da organização. Para tal, foi escolhida a regra de escolha de Borda. Nela, cada 
decisor atribui a nota 0 para a última alternativa no seu ranking, 1 para a penúltima, ..., e m-1 para a sua alternativa preferida. As notas de cada alternativa, para cada decisor, são somadas e aquela que obtiver a maior soma é considerada a melhor alternativa.

O processo de tomada de decisão, ilustrado na Figura 2, é iniciado sempre que houver a necessidade de alterar um processo padrão da organização ou um processo definido para um projeto em execução. É composto por três atividades, descritas nas seções a seguir.

\subsubsection{Estruturação}

Nesta atividade, composta por duas tarefas (Tabela 1), o grupo de decisão define as possíveis alternativas de processo (incluindo a versão original do processo), bem como os critérios que serão utilizados para avaliar cada alternativa.

Tabela 1. Atividade Estruturação

\begin{tabular}{|l|l|}
\hline Tarefa: & Definir alternativas de processo \\
\hline Descrição: & $\begin{array}{l}\text { Nesta tarefa, as possíveis alternativas de processo são definidas. O processo original também } \\
\text { é considerado uma das alternativas. }\end{array}$ \\
\hline Tarefa: & Definir critérios para a avaliação \\
\hline Descrição: & $\begin{array}{l}\text { Nesta tarefa, os critérios que serão adotados para comparar as alternativas são definidos, } \\
\text { juntamente com seus desempenhos esperados e objetivos organizacionais de desempenho. } \\
\text { Cada critério deve ter a orientação da sua escala definida para que seja possível diferenciar } \\
\text { critérios com escala orientada positivamente de critérios com escala orientada negativamente. }\end{array}$ \\
\hline
\end{tabular}

\subsubsection{Avaliação}

A atividade Avaliação é composta por sete tarefas (Tabela 2), que, quando executadas, geram o modelo de decisão para o problema a ser tratado. As tarefas que compõem esta atividade são executadas individualmente, por cada participante do grupo de decisão.

\section{Tabela 2. Atividade Avaliação}

\begin{tabular}{|l|l|}
\hline Tarefa: & Estabelecer pesos para os critérios \\
\hline Descrição: & $\begin{array}{l}\text { Nesta tarefa, cada decisor define, por meio de pesos, a importância relativa de cada critério } \\
\text { para o problema de decisão a ser tratado. }\end{array}$ \\
\hline Tarefa: & Estabelecer desempenhos esperados para cada critério \\
\hline Descrição: & $\begin{array}{l}\text { Nesta tarefa, cada decisor estabelecerá o desempenho esperado para cada critério, conside- } \\
\text { rando cada uma das alternativas de processo. }\end{array}$ \\
\hline Tarefa: & Definir matriz de decisão normalizada \\
\hline Descrição: & $\begin{array}{l}\text { Tendo definidos os critérios, seus respectivos pesos e os desempenhos esperados para as } \\
\text { alternativas em cada critério, é possível definir a matriz de decisão. Nesta matriz, os desem- } \\
\text { penhos das alternativas em cada critério são normalizados e multiplicados pelo seu peso. }\end{array}$ \\
\hline Tarefa: & Identificar solução ideal e solução indesejada \\
\hline Descrição: & $\begin{array}{l}\text { Esta tarefa tem como objetivo a identificação da solução ideal e da solução indesejada. Vale } \\
\text { ressaltar que estes piores desempenhos devem observar a orientação da escala de cada crité- } \\
\text { rio. Para critérios de escala com orientação negativa, o pior desempenho é o maior valor e, } \\
\text { para critérios de escala com orientação positiva, o pior desempenho é o menor valor. }\end{array}$ \\
\hline Tarefa: & Calcular distâncias de cada alternativa à solução ideal e à solução indesejada \\
\hline Descrição: & $\begin{array}{l}\text { Nesta tarefa é verificada o quão próxima uma alternativa de processo está da solução ideal e } \\
\text { o quão distante ela se encontra da solução indesejada. Para tal, é utilizada a Distância Eucli- } \\
\text { diana de cada alternativa de processo para a solução ideal e para a solução indesejada. }\end{array}$ \\
\hline Tarefa: & Calcular a proximidade relativa de cada alternativa com a solução ideal \\
\hline Descrição: & $\begin{array}{l}\text { Nesta tarefa é verificado o quão similar é à solução ideal de cada alternativa de processo } \\
\text { definida (incluindo o processo atual). Para isto, é calculado um índice de similaridade que } \\
\text { combina a proximidade com a solução ideal e o afastamento da solução indesejada. }\end{array}$ \\
\hline
\end{tabular}




\begin{tabular}{|l|l|}
\hline Tarefa: & Definir ordem de preferência individual das alternativas de processo \\
\hline Descrição: & $\begin{array}{l}\text { Esta tarefa conclui a atividade Avaliação. Com base no índice de similaridade com a solução } \\
\text { ideal, calculado para cada alternativa de processo na tarefa anterior, é elaborada uma lista, } \\
\text { para cada decisor, contendo a ordem de preferência das alternativas de processos. }\end{array}$ \\
\hline
\end{tabular}

\subsubsection{Recomendações}

A atividade Recomendações é composta por três tarefas, descritas na Tabela 3.

\section{Tabela 3. Atividade Recomendações}

\begin{tabular}{|c|c|}
\hline Tarefa: & Executar análise de sensibilidade \\
\hline Descrição: & $\begin{array}{l}\text { Esta tarefa é executada após a execução do método AMD. Nela, são definidos cenários de } \\
\text { variações dos pesos de cada critério, considerando que os demais critérios permanecem com } \\
\text { seus pesos fixos. No primeiro cenário, é atribuído a um determinado critério um peso de } \\
\text { valor } 0 \text {. No cenário seguinte, o peso passa a ser } 0.1 \text { e assim sucessivamente até alcançar o } \\
\text { valor } 1 \text {. Para cada variação do peso, o Índice de Similaridade é recalculado. A partir dos } \\
\text { valores obtidos para os Índices de Similaridade, são gerados gráficos que demonstram o } \\
\text { comportamento da ordem de preferência das alternativas ao longo da variação do peso do } \\
\text { critério. Estes gráficos têm a função de auxiliar o decisor a identificar o critério mais crítico, } \\
\text { ou seja, aquele cujo valor do peso que causa alteração no resultado do processo de decisão } \\
\text { está mais próximo do valor originalmente a ele atribuído. Critérios críticos devem ser anali- } \\
\text { sados com mais cuidado. }\end{array}$ \\
\hline Tarefa: & Consolidar preferências individuais \\
\hline Descrição: & $\begin{array}{l}\text { Nesta tarefa, cada decisor recebe um peso e todos os resultados individuais são consolidados } \\
\text { por meio da regra de escolha de Borda, gerando uma nova lista de preferências das alternati- } \\
\text { vas. Esta lista consolidada é considerada a decisão do grupo. }\end{array}$ \\
\hline Tarefa: & Escolher a melhor alternativa de processo \\
\hline Descrição: & $\begin{array}{l}\text { Nesta tarefa, o grupo de decisão escolhe, com base na lista de preferência coletiva das alter- } \\
\text { nativas de processo, o melhor processo para a organização ou para o projeto. }\end{array}$ \\
\hline
\end{tabular}

\subsection{Processo da Abordagem}

Com o intuito de facilitar o entendimento da proposta como um todo, foi definido um processo composto por 13 tarefas distribuídas em 3 atividades, como ilustra a Figura 3.

\subsubsection{Avaliar Impacto da Alteração no Processo Padrão}

Alterações em processos padrão podem fazer com que ele apresente um desempenho pior do que o processo padrão original [Raffo, 2005], o que torna necessário avaliar previamente os efeitos destas mudanças. Nesta proposta, a avaliação do impacto da alteração no processo padrão dar-se-á por meio da comparação de alternativas de processos padrão, incluindo o processo padrão original, com relação a critérios que podem estar associados, por exemplo, aos objetivos estratégicos organizacionais ou objetivos específicos de projeto. Esta atividade é composta por quatro tarefas, apresentadas na Tabela 4.

\section{Tabela 4. Atividade Avaliar Impacto da Alteração no Processo Padrão}

\begin{tabular}{|l|l|}
\hline Tarefa: & Analisar necessidades de melhoria \\
\hline Descrição: & $\begin{array}{l}\text { Esta tarefa tem por objetivo analisar as necessidades de melhoria registradas no Repositório } \\
\text { de Necessidades de Melhoria, selecionar as que devem ser implementadas e definir a ordem } \\
\text { de prioridade. As necessidades que serão implementadas devem passar da situação "aberta" } \\
\text { para "em planejamento". }\end{array}$ \\
\hline Tarefa: & Definir itens de ação e planejar melhoria \\
\hline Descrição: & $\begin{array}{l}\text { Após a análise das necessidades de melhoria, são definidos os itens de ação para cada proces- } \\
\text { so padrão correspondente. Um plano de ação para implementação das melhorias é, então, } \\
\text { elaborado. Este planejamento deve definir também quais indicadores serão utilizados para } \\
\text { monitorar os efeitos das melhorias, a periodicidade da monitoração e a quantidade de monito- }\end{array}$ \\
\hline
\end{tabular}




\begin{tabular}{|c|c|}
\hline & rações necessárias para que seja possível avaliar os efeitos das melhorias. \\
\hline Tarefa: & Executar processo de tomada de decisão com TOPSIS \\
\hline Descrição: & $\begin{array}{l}\text { Nesta tarefa, as alternativas de processo padrão, incluindo o processo padrão atual, são com- } \\
\text { paradas. Quando o processo atual é considerado o mais adequado, volta-se para a atividade } \\
\text { anterior para definir novos itens de ação ou adia-se a melhoria. A avaliação das alternativas } \\
\text { de processo é apoiada pelo processo de tomada de decisão com TOPSIS, definido na seção } \\
\text { 3.1. Se o resultado da tomada de decisão definir que o processo atual é o mais apropriado, o } \\
\text { Grupo de Processos pode optar ou por definir novos itens de ação ou adiar a melhoria. Deci- } \\
\text { dindo pelo adiamento, a situação da necessidade de melhoria deve retornar para "aberta". }\end{array}$ \\
\hline Tarefa: & Alterar processo padrão \\
\hline Descrição: & $\begin{array}{l}\text { Esta tarefa é executada quando se decide alterar o processo padrão. Seu objetivo é alterar o } \\
\text { processo padrão com base na alternativa de processo escolhida. Inicialmente, este novo pro- } \\
\text { cesso padrão será rotulado como "em avaliação". Uma vez alterado, o novo processo padrão } \\
\text { deve ser utilizado em novos projetos e, se possível, nos projetos em execução. Neste momen- } \\
\text { to, a versão anterior do processo padrão passa a ter a situação "descontinuado". Além disso, } \\
\text { as necessidades implementadas devem passar para a situação "em avaliação". }\end{array}$ \\
\hline
\end{tabular}

\subsubsection{Avaliar Impacto da Alteração nos Processos Definidos em Execução}

Uma vez que melhorias são implementadas, os novos projetos devem fazer uso do novo processo padrão da organização. Também é importante avaliar a viabilidade de se utilizar este novo processo padrão nos projetos em execução que tiveram seus processos definidos com base na versão anterior do processo padrão.

Diante deste contexto, a atividade "Avaliar impacto da alteração nos processos definidos em execução" objetiva identificar os processos definidos para os projetos em execução que adotaram a versão anterior do processo padrão. Uma vez identificados os processos definidos em execução, para cada projeto correspondente, deve-se avaliar o impacto da alteração no seu processo definido para decidir se pode ser vantajosa ou não. Se o resultado da avaliação revelar que é vantajoso para o projeto alterar seu processo definido para que ele seja adaptado a partir do novo processo padrão, o processo definido é alterado e o projeto passa a executar essa nova versão. Caso contrário, o projeto segue executando seu processo definido com base na versão anterior do processo padrão. Esta atividade é composta de três tarefas, descritas na Tabela 5.

Tabela 5. Atividade Avaliar Impacto da Alteração nos Processos Definidos em Execução

\begin{tabular}{|l|l|}
\hline Tarefa: & Selecionar processos em execução, cujos projetos podem ser beneficiados pela mudança \\
\hline Descrição: & $\begin{array}{l}\text { Esta tarefa tem como objetivo identificar, dentre os projetos em execução que tiveram seus } \\
\text { processos definidos com base na versão anterior do processo padrão, aqueles que podem ser } \\
\text { beneficiados pela mudança. }\end{array}$ \\
\hline Tarefa: & Executar processo de tomada de decisão com TOPSIS para cada projeto selecionado \\
\hline Descrição: & $\begin{array}{l}\text { O objetivo desta tarefa é, para cada processo definido selecionado na atividade anterior, com- } \\
\text { parar as alternativas de processo definido (incluindo o processo definido atual), com relação a } \\
\text { critérios previamente especificados, via mecanismo de apoio à tomada de decisão (seção 3.1). }\end{array}$ \\
\hline Tarefa: & $\begin{array}{l}\text { Alterar processos dos projetos onde a adoção do novo processo definido foi considerada } \\
\text { vantajosa }\end{array}$ \\
\hline Descrição: & $\begin{array}{l}\text { Esta tarefa é executada quando, pelo menos, um processo definido em execução for selecio- } \\
\text { nado para ser alterado com base no novo processo padrão. Após a alteração, o projeto passará } \\
\text { a executar este novo processo definido. }\end{array}$ \\
\hline
\end{tabular}

\subsubsection{Monitorar Efeitos da Alteração no Processo Padrão}

Esta atividade tem como objetivo a monitoração e o registro dos efeitos das alterações no processo padrão a partir dos indicadores definidos na tarefa "Definir itens de ação e planejar melhoria”. A Tabela 6 detalha as seis tarefas que compõem esta atividade. 


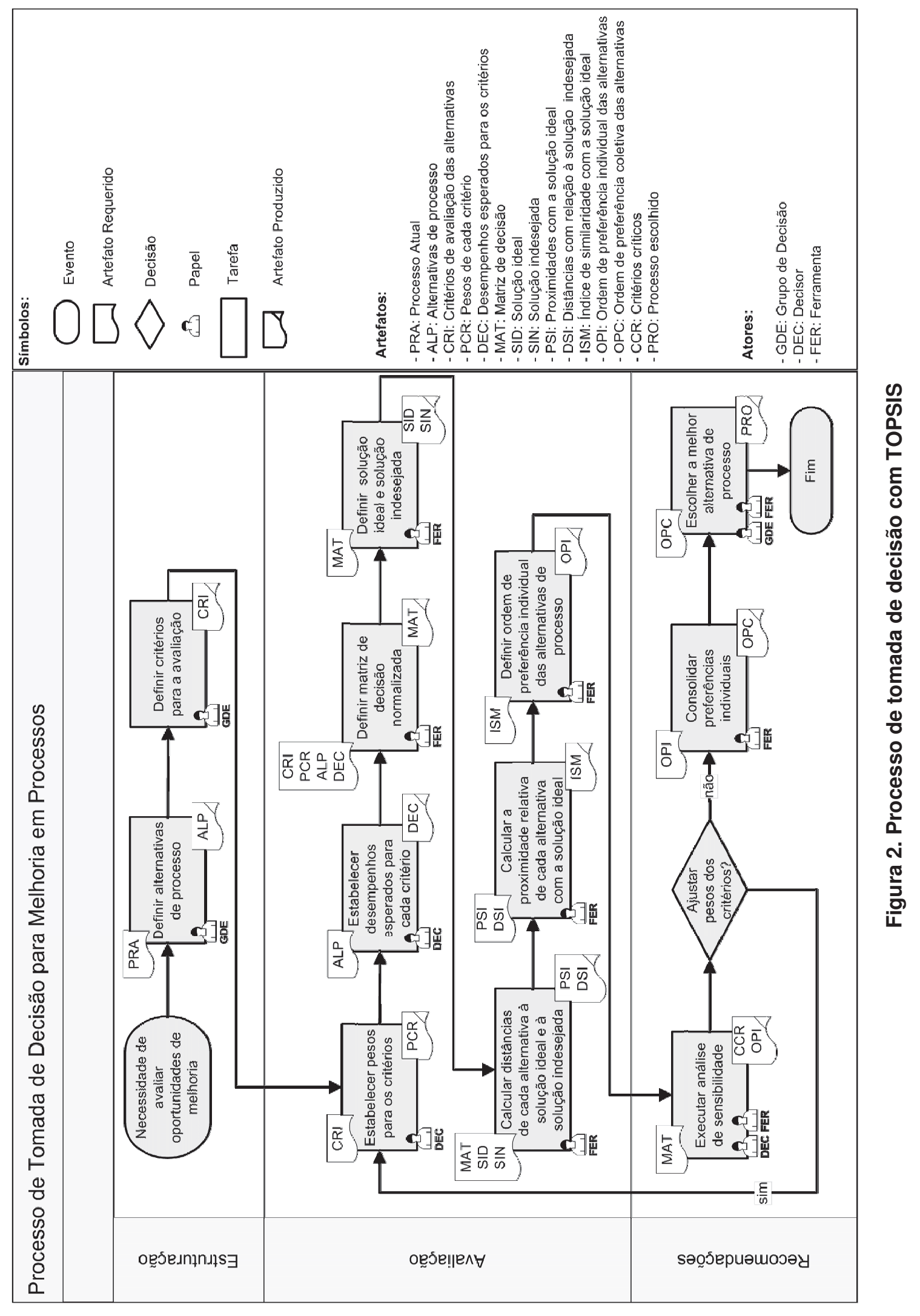




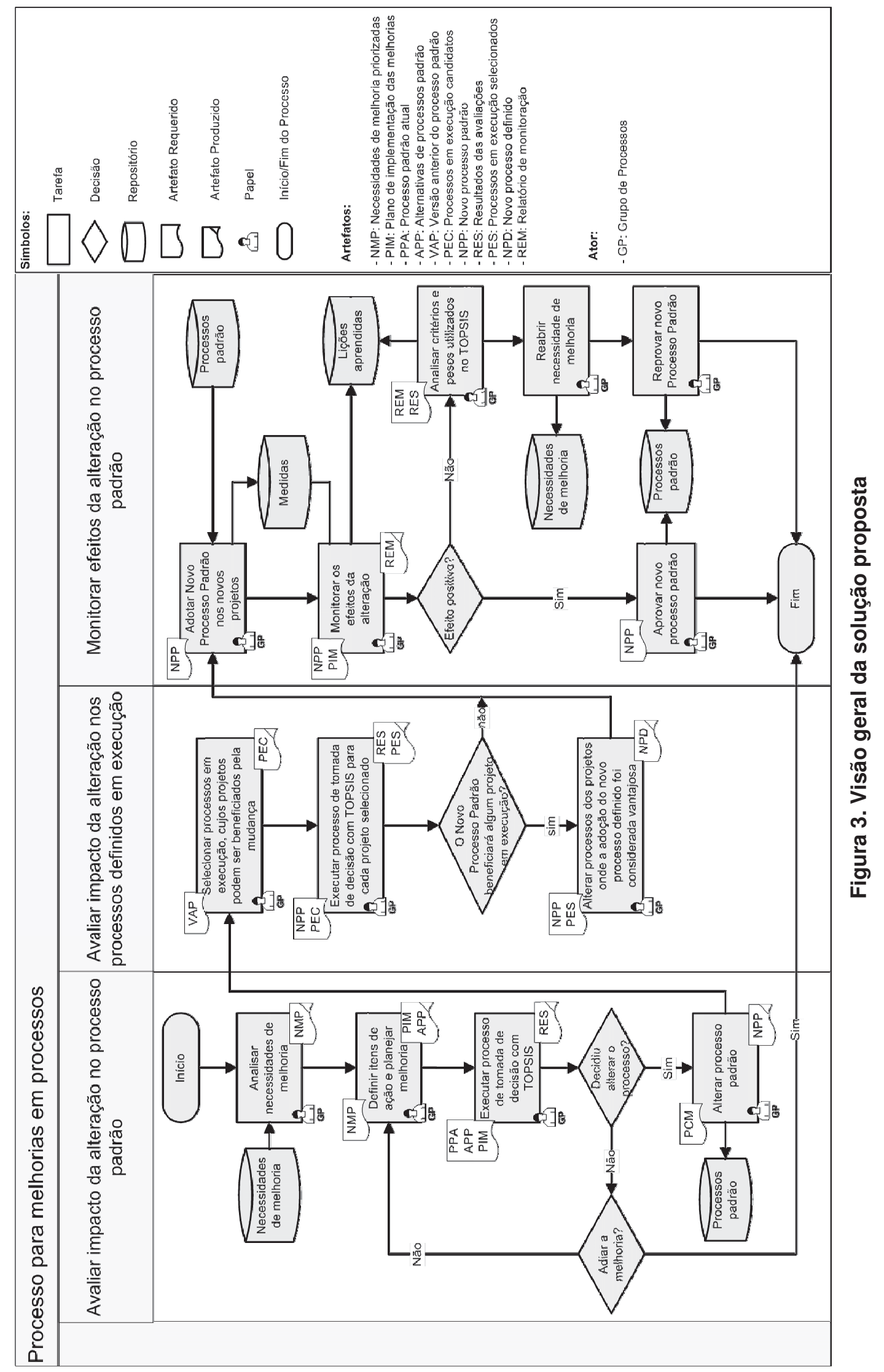


Tabela 6. Atividade Monitorar Efeitos da Alteração no Processo Padrão

\begin{tabular}{|c|c|}
\hline Tarefa: & Adotar Novo Processo Padrão nos novos projetos \\
\hline Descrição: & $\begin{array}{l}\text { Esta atividade é executada sempre que o processo padrão é alterado. Os novos projetos de- } \\
\text { vem ter seus processos definidos com base neste novo processo padrão. }\end{array}$ \\
\hline Tarefa: & Monitorar os efeitos da alteração \\
\hline Descrição: & $\begin{array}{l}\text { Esta atividade é executada de acordo com a periodicidade definida no plano de implementa- } \\
\text { ção das melhorias. O objetivo desta tarefa é monitorar os efeitos reais da alteração do proces- } \\
\text { so padrão, a partir dos indicadores definidos no plano de implementação das melhorias. Esta } \\
\text { monitoração serve para prevenir os projetos de impactos negativos por conta do novo proces- } \\
\text { so definido com base no novo processo padrão. }\end{array}$ \\
\hline Tarefa: & Analisar critérios e pesos utilizados no modelo de decisão \\
\hline Descrição: & $\begin{array}{l}\text { Esta tarefa deve ser executada sempre que os efeitos da execução do novo processo padrão } \\
\text { forem negativos. Os critérios e pesos definidos devem ser revisados para identificar possíveis } \\
\text { falhas na construção do modelo de decisão. Caso sejam identificadas falhas, estas devem ser } \\
\text { registradas como lições aprendidas de modo a evitar que sejam repetidas. }\end{array}$ \\
\hline Tarefa: & Reabrir necessidade de melhoria \\
\hline Descrição: & $\begin{array}{l}\text { Esta tarefa deve ser executada sempre que os efeitos da execução do novo processo padrão } \\
\text { forem negativos. Como o novo processo padrão não atingiu os objetivos esperados, a situa- } \\
\text { ção da necessidade de melhoria deve ser alterada para "aberta" para que novos itens de ação } \\
\text { sejam planejados para o próximo ciclo de melhorias. }\end{array}$ \\
\hline Tarefa: & Reprovar novo Processo Padrão \\
\hline Descrição: & $\begin{array}{l}\text { Esta tarefa deve ser executada quando os efeitos da execução do novo processo padrão forem } \\
\text { negativos. A situação do novo processo padrão deve ser alterada para "reprovado". }\end{array}$ \\
\hline Tarefa: & Aprovar novo Processo Padrão \\
\hline Descrição: & $\begin{array}{l}\text { Esta tarefa deve ser executada sempre que os efeitos da execução do novo processo padrão } \\
\text { mostrarem-se positivos. A sua situação deve ser alterada para "aprovado". }\end{array}$ \\
\hline
\end{tabular}

\section{Avaliação}

A proposta deste trabalho foi avaliada por meio da execução de três estudos de caso na indústria. Baseado no modelo de aceitação de tecnologia (Technology Acceptance Model - TAM) [Davis, 1989], a proposta foi avaliada com relação aos dois principais determinantes: (i) utilidade percebida: grau em que uma pessoa acredita que o uso da tecnologia pode melhorar o seu desempenho; e (ii) facilidade percebida: grau em que uma pessoa acredita que o uso de uma tecnologia será livre de esforço mental e físico. Diante deste contexto, o objetivo do estudo experimental, segundo o paradigma GQM [Basili e Rombach, 1988], foi definido como mostra Quadro 2:

\section{Quadro 2. Objetivo do estudo experimental}

Analisar o uso da solução proposta para avaliação de alternativas de processo e seleção do melhor processo.

Com o propósito de caracterizar

Com relação à utilidade percebida e à facilidade de uso percebida

Do ponto de vista de membros do grupo de processos

No contexto de melhoria de processos em uma organização de desenvolvimento de software.

Foram conduzidos três estudos de caso, cada um em uma organização de desenvolvimento de software diferente, avaliando processos distintos. Cada estudo de caso foi executado em três etapas. Na primeira etapa, os participantes definiram as alterações a serem realizadas no processo e escolheram o processo que consideravam mais apropriado. Em seguida, foi solicitado que preenchessem um formulário pré-execução, cujo objetivo era caracterizar o participante quanto à sua experiência em melhoria de processos. A segunda etapa consistiu em uma apresentação do processo da proposta, treinamento do mecanismo de apoio à tomada de decisão e, por fim, da utilização do mecanismo 
para gerar os modelos de decisão individual. Nos estudos executados por mais de um participante, os critérios de comparação, desempenhos atuais do processo padrão e objetivos organizacionais de desempenho foram definidos em consenso. No final, os modelos de decisão individual foram consolidados para gerar as recomendações coletivas. A terceira etapa visou avaliar, a partir de um formulário pós-execução, a percepção de cada participante quanto à facilidade e à utilidade da proposta.

As ameaças relacionadas a este estudo seguem os tipos de ameaças apresentados em [WÖHLIN et al., 2000] e estão descritas no Quadro 3.

\section{Quadro 3. Ameaças à validade}

Validade interna. A medição do tempo poderia influenciar o resultado do estudo. Para mitigar esta ameaça, o tempo foi medido pela pesquisadora. Além disso, foi acrescentada ao formulário pósexecução uma pergunta questionando se o tempo despendido na execução da proposta foi factível ou não. Outra ameaça que precisa ser considerada é que as opiniões dos participantes podem ter sido influenciadas pela preocupação em não prejudicar os resultados do trabalho.

Validade externa. Este tipo de ameaça foi reduzido pelo fato de os participantes terem alto nível de experiência em melhorias de processos e pelo fato de os estudos de casos terem sido conduzidos em processos reais, com problemas reais e em contextos distintos.

Validade de construção (ou Constructo). Uma ameaça à validade de construção poderia ser a escolha dos indicadores. No entanto, a ameaça foi minimizada com a adoção dos indicadores sugeridos pelo TAM [Davis, 1989].

Validade de conclusão. A principal ameaça a este tipo de validade é o tamanho da amostra, cujo número de data points não tem significância estatística. Por esta razão, os resultados do estudo não podem ser considerados conclusivos, mas apenas indícios.

\subsection{Execução dos Estudos de Caso}

\subsubsection{Organização A}

A Organização A foi avaliada no nível C do MR-MPS-SW. O dois participantes são implementadores e avaliadores MR-MPS-SW, com mais de 9 anos de experiência em melhorias de processos. Os participantes selecionaram duas melhorias no processo padrão de desenvolvimento, definiram uma alternativa de processo e, com base em suas experiências, decidiram que o novo processo com estas duas alterações seria mais adequado para a Organização A do que o processo padrão atual. Em seguida, eles executaram o processo de tomada de decisão. Após a consolidação dos modelos de decisão individual, a recomendação foi que o novo processo com as melhorias planejadas seria mais apropriado para a Organização A, coincidindo com a opinião dos participantes.

O passo seguinte foi a identificação dos projetos em execução, que poderiam ser beneficiados pela mudança. Neste momento, apenas um dos participantes executou o processo, pois estava diretamente envolvido com os projetos em execução. A recomendação gerada foi a de que poderia ser vantajoso para o projeto adotar o novo processo padrão com as melhorias planejadas. O participante concordou com o resultado.

\subsubsection{Organização B}

A Organização B foi recentemente avaliada no nível C do MR-MPS-SW. O estudo de caso contou com a participação de um único membro do Grupo de Processos. O participante tinha mais de 6 anos de experiência em melhorias de processos. O participante selecionou duas melhorias para o processo padrão de Verificação, definiu três alternativas de processo e escolheu uma das alternativas como a mais adequada para a sua orga- 
nização. A recomendação final após a execução do processo de tomada de decisão foi que não alterasse o processo padrão. A alternativa de processo escolhida pelo participante foi considerada a segunda melhor opção de processo para a Organização B. Embora trazendo um resultado divergente da sua opinião inicial, o participante concordou que as oportunidades de melhoria avaliadas requeriam uma melhor preparação da organização antes de serem implementadas.

Como a decisão tomada foi a de manter o processo padrão atual, não houve necessidade de avaliar os projetos em execução.

\subsubsection{Organização C}

A Organização C foi avaliada no nível G do MR-MPS-SW. Os dois participantes do estudo eram implementadores e avaliadores do MR-MPS-SW, com mais de 6 anos de experiência em melhorias de processos. Os participantes planejaram a implementação de uma única melhoria no processo padrão de desenvolvimento, definiram duas alternativas de processo e decidiram que o processo atual ainda seria o mais adequado para a organização. A execução do processo de tomada de decisão recomendou uma das alternativas de processo definidas. Os participantes concordaram com a recomendação fornecida e acabaram acatando as reivindicações dos colaboradores.

Como a Organização C não possuía projetos em execução que estivessem utilizando o processo padrão a ser alterado, não foi possível avaliar a viabilidade de adotar o novo processo padrão nos projetos em execução.

\subsection{Resultados}

Os resultados obtidos com relação à percepção dos cinco participantes sobre a utilidade da proposta podem ser observados na Figura 4.
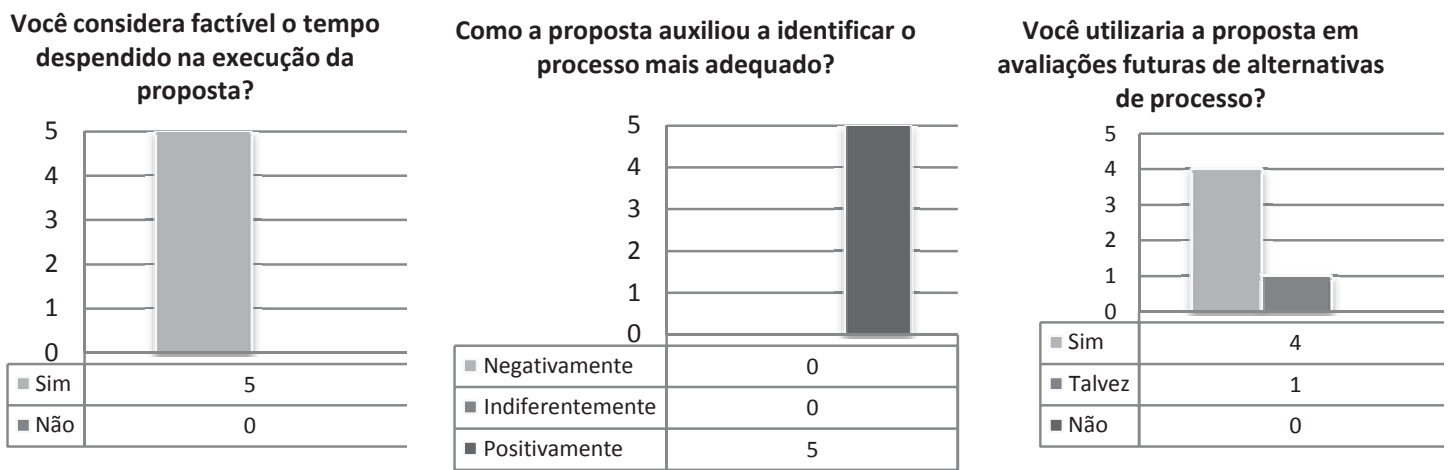

Figura 4. Resultados quanto à utilidade percebida

Com relação à facilidade de uso percebida pelos participantes do estudo, três participantes afirmaram que o grau de dificuldade é fácil e dois participantes afirmaram que o grau de dificuldade é mediano (Figura 5). As justificativas para o grau de dificuldade mediano foram a necessidade de auxílio para a definição da orientação dos critérios (positiva e negativa) e a definição consensual dos critérios de avaliação. Diante das informações apresentadas, é possível afirmar que os participantes do estudo consideraram a proposta útil e fácil de utilizar.

Vale destacar que, em dois dos estudos de caso, a proposta fez com que os participantes mudassem suas opiniões iniciais sobre qual processo seria o mais apropriado 
para a organização. Foi percebida uma forte tendência das pessoas considerarem apenas os aspectos (critérios) nos quais o processo considerado inicialmente como o mais adequado apresentava melhor desempenho. Isso pode ser um grande limitador quando a tomada de decisão não é estruturada, podendo levar a escolhas equivocadas.

Devido à pequena quantidade de participantes do estudo, o que pode ser afirmado, por enquanto, é que há indícios de que a proposta auxilia a avaliação prévia alternativas de processo antes que as ações planejadas sejam de fato implementadas.

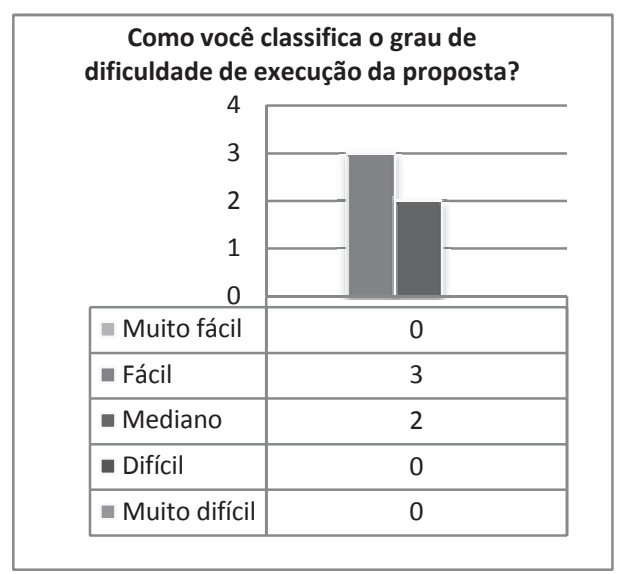

Figura 5. Resultados quanto à facilidade percebida

\section{Considerações Finais}

Continuamente, as organizações de desenvolvimento de software precisam melhorar seus processos padrão. Uma vez implementadas melhorias, é interessante que os projetos em execução sejam avaliados quanto aos benefícios que podem ser obtidos caso as melhorias no processo padrão sejam estendidas aos seus processos definidos.

Poucas abordagens que lidam com alterações em processos durante a execução do projeto se preocupam em avaliar previamente os efeitos da mudança. Além disso, as que tratam este problema, possuem limitações ou quanto à exigência de dados históricos de execuções dos processos ou com relação aos parâmetros analisados ou pela necessidade de um conhecimento específico, além do papel que o decisor assume na organização. Com o intuito de preencher esta lacuna, foi apresentada uma proposta para apoiar a avaliação prévia de melhorias em processos definidos para um projeto em execução, baseada no método multicritério de apoio à tomada de decisão TOPSIS.

A proposta foi avaliada por meio de três estudos de caso, que revelaram indícios de que ela pode ser útil e fácil de utilizar.

Como trabalhos futuros pretende-se: (i) estudar uma maneira de conduzir o grupo de decisão a identificar mais facilmente os melhores critérios e; (ii) estender a Análise de Sensibilidade para avaliar em tempo hábil o quão sensível é o modelo gerado a pequenas variações nos desempenhos atribuídos aos critérios.

\section{Referências}

Al-Shemmeri, T., Al-Kloub, B. e Pearman, A. (1997). "Model choice in multicriteria decision aid." European Journal of Operational Research 97(3): 550-560.

Basili, V. R. e Rombach, H. D. (1988). "The TAME project: towards improvement- 
oriented software environments." Software Engineering, IEEE Transactions on 14(6): 758-773.

Beydeda, S. e Gruhn, V. (2004). "Dynamic evolution of software processes to evolve software systems during their development." Software Process Improvement and Practice 9(4): 229-238.

Brans, J. P. (2002). "Ethics and decision." European Journal of Operational Research 136(2): 340-352.

Cabral, M. (2012). Avaliação de Melhorias em Processos de Software Durante a Execução de um Projeto. Programa de Engenharia de Sistemas e Computação. Rio de Janeiro, Universidade Federal do Rio de Janeiro. M. Sc.

Davis, F. D. (1989). A technology acceptance model for empirically testing new enduser information systems : theory and results. Sloan School of Management. Massachusetts, Massachusetts Institute of Technology.

Forneck, M. (2008). Análise Multicirterial das Preferências de Gestores e das Decisões nas Operações Logísticas de uma Empresa do setor Químico. São Leopoldo, UNISINOS. Ms.C.: 109.

Guitouni, A. e Martel, J.-M. (1998). "Tentative guidelines to help choosing an appropriate MCDA method." European Journal of Operational Research 109(2): 501-521.

Hair, J. F., Anderson, R. E., Tatham, R. L. e Black, W. C. (2007). Análise Multivariada de Dados, Bookman.

Liou, J. J. H., Hsu, C., Yeh, W. e Lin, R. (2011). "Using a modified grey relation method for improving airline service quality." Tourism Management 32(6): 1381-1388.

Malakooti, B. (1988). "A decision support system and a heuristic interactive approach for solving discrete multiple criteria problems." Systems, Man and Cybernetics, IEEE Transactions on 18(2): 273-284.

Park, S., Choi, K., Yoon, K. A. e Bae, D. (2007). Deriving Software Process Simulation Model from SPEM-based Software Process Model. Software Engineering Conference, 2007. APSEC 2007. 14th Asia-Pacific. Aichi: 382-389.

Raffo, D., Spehar, G. e Nayak, U. (2003). Generalized Simulation Models: What, Why and How? ProSim 2003, Oregon.

Raffo, D. M. (2005). "Software project management using PROMPT: A hybrid metrics, modeling and utility framework." Information and Software Technology 47(15): 1009-1017.

SEI (2010). CMMI for Development (CMMI-DEV), Version 1.3., Pittsburgh, PA: Software Engineering Institute, Carnegie Mellon University.

SOFTEX. (2012). "MPS.BR - Guia Geral." Disponível em: www.softex.br.

WÖHLIN, C., RUNESON, P., HÖST, M., OHLSSON, M., REGNELL, B. e WESSLÉN, A. (2000). Experimentation in Software Engineering: An Introduction. Norwell, MA, USA, Kluwer Academic Publishers.

Yoon, K. P. e Hwang, C.-L. (1995). Multiple Attribute Decision Making: an Introduction. Thousand Oaks, California, SAGE Publications, Inc. 